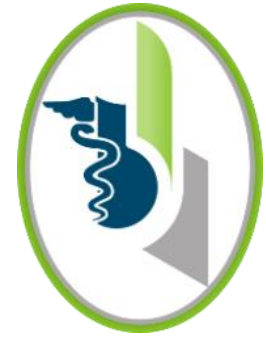

ACCESO $\odot$ ABIERTO

Para citaciones: Espitia, F. (2021). Indicaciones de la drospirenona más allá de la anticoncepción. Revista Ciencias Biomédicas, 10(2), 120-128. https://doi.org/10.32997/rcb-2021-3372

Recibido: 1 de febrero de 2021 Aprobado: 8 de marzo de 2021

Autor de correspondencia: Franklin José Espitia De La Hoz espitiafranklin@hotmail.com

Editor: Inés Benedetti. Universidad de Cartagena-Colombia.

\section{Indicaciones de la drospirenona más allá de la anticoncepción}

\author{
Indications of drospirenone beyond contraception \\ Franklin José Espitia De La Hoz ${ }^{1}$ (D) \\ ${ }^{1}$ Dirección científica Hathor Clínica Sexológica, Armenia, Colombia.
}

\section{RESUMEN}

Introducción: la drospirenona es una progestina de tercera generación, derivada de la espironolactona; la cual goza de actividad anti-mineralocorticoide y antiandrogénica, además de una alta eficacia anticonceptiva. El objetivo de esta revisión consistió en evaluar la efectividad y seguridad de la drospirenona, más allá de sus efectos anticonceptivos.

Métodos: se hizo una revisión sistemática de la literatura en las bases de datos electrónicas (Medline vía PubMed, Central, CINAHL, Cochrane Database of Systematic Reviews (plataforma Wiley), entre otras), a través de términos de búsqueda libres y estandarizados. Los desenlaces evaluados incluyeron tratamiento de la endometriosis, síndrome de ovario poliquístico, sangrado uterino disfuncional, síndrome premenstrual y terapia de reemplazo hormonal, así como los efectos adversos. La búsqueda se limitó a artículos y revisiones bibliográficas publicadas a partir de 1990 hasta el 2020, en inglés y español.

Resultados: se incluyeron 48 publicaciones. La drospirenona demostró ser eficaz y segura en el tratamiento de condiciones ginecológicas frecuentes como: la endometriosis, síndrome de ovario poliquístico (SOP), sangrado uterino disfuncional, síndrome premenstrual y en la terapia de reemplazo hormonal. Se observó una baja frecuencia de efectos adversos.

Conclusiones: la drospirenona es eficaz y segura para el tratamiento de algunas afecciones ginecológicas de frecuente consulta en la práctica diaria, con ventajas adicionales en su régimen 24/4, además de escasa presencia de efectos adversos. Se requiere mayor evidencia más allá de la anticoncepción, para promover su recomendación e indicaciones en la práctica clínica.

Palabras Clave: Anticonceptivos; Endometriosis; Hiperandrogenismo; Menopausia; Progestinas; Síndrome Premenstrual.

\section{ABSTRACT}

Introduction: Drospirenone is a third generation progestin, derived from spironolactone; which has anti-mineralocorticoid and anti-androgenic activity, as well as high contraceptive efficacy. The objective of this review was to assess the effectiveness and safety of drospirenone, beyond its contraceptive effects. 
Methods: a systematic review of the literature was made in electronic databases (Medline via PubMed, Central, CINAHL, Cochrane Database of Systematic Reviews (Wiley platform), among others), through free and standardized search terms. The outcomes evaluated included treatment of endometriosis, polycystic ovary syndrome, dysfunctional uterine bleeding, premenstrual syndrome, and hormone replacement therapy, as well as adverse effects. The search was limited to articles and bibliographic reviews published from 1990 to 2020, in English and Spanish.

Results: 48 publications were included. Drospirenone has been shown to be effective and safe in the treatment of common gynecological conditions such as: endometriosis, polycystic ovary syndrome (PCOS), dysfunctional uterine bleeding, premenstrual syndrome, and in hormone replacement therapy. A low frequency of adverse effects was observed.

Conclusions: Drospirenone is effective and safe for the treatment of some gynecological conditions that are frequently consulted in daily practice, with additional advantages in its 24/4 regimen, in addition to the scarce presence of adverse effects. More evidence beyond contraception is required to promote its recommendation and indications in clinical practice.

Keywords: Contraceptive Agents; Endometriosis; Hyperandrogenism; Menopause; Progestins; Premenstrual Syndrome.

\section{INTRODUCCIÓN}

La drospirenona (DRSP) es una progestina, estructural y funcionalmente análoga a la espironolactona; la cual exhibe efectos tanto antimineralocorticoides como anti-androgénicos; tiene una biodisponibilidad del $76 \%$ a $80 \%$, aproximadamente el $20 \%$ se excreta a través de las heces y el $45 \%$ por el sistema renal. Su vida media es de 30 a 35 horas. Se estima que es 8 veces más potente que la espironolactona ( $3 \mathrm{mg}$ de $\mathrm{DRSP}=25$ mg de espironolactona); además carece de metabolitos activos, una característica que la distingue de la espironolactona $(1,2)$.

Las progestinas sintéticas se han utilizado ampliamente como anticonceptivos, también para tratar la endometriosis, el cáncer de endometrio, el síndrome de ovario poliquístico (SOP), el sangrado uterino disfuncional, el síndrome premenstrual y en la terapia de reemplazo hormonal (3-6); en parte gracias a que causan decidualización y atrofia del endometrio $(7,8)$.
Al utilizar la drospirenona, en el mismo rango de dosis orales $(0,5-4,0 \mathrm{mg}$ por día), se ha observado transformación endometrial, inhibición del desarrollo folicular y de la ovulación, al suprimir la hormona luteinizante (LH); además aumenta la viscosidad del moco cervical y reduce la producción de hormona androgénica ovárica, así como de presentar efectos anti-mineralocorticoides (natriuréticos: reduce la presión arterial y la excreción de potasio), con efectos antiandrogénicos leves $(9,10)$.

La drospirenona es una progestina ampliamente estudiada como anticonceptivo en combinación con un estrógeno; sin embargo, el régimen 24/4 (4 mg durante 24 días seguida de placebo durante 4 días) ha demostrado eficacia anticonceptiva con un índice de Pearl de 0,51 (IC95\%: 0,11-1,49) (10, 11); eficacia anticonceptiva que se mantiene, incluso cuando se ha retrasado la ingesta de la píldora durante 24 horas (12). El régimen de $4 \mathrm{mg} 24 / 4$ proporciona una eficacia anticonceptiva similar a la de muchas combinaciones orales, con un buen perfil de seguridad y un control favorable del ciclo $(11,13)$. 
No obstante, al revisar diferentes estudios, disponibles en la literatura, se ha encontrado que las progestinas tienen indicaciones que sobrepasan sus efectos anticonceptivos; y ante la creciente necesidad de píldoras anticonceptivas de progestina sola, debido al riesgo cardiovascular asociado con los estrógenos en las píldoras anticonceptivas orales combinadas $(14,15)$, han motivado la presente revisión, cuyo objetivo consistió en evaluar la efectividad y seguridad de la drospirenona, en indicaciones más allá de sus efectos anticonceptivos.

\section{MÉTODOS}

Se realizó una búsqueda de la literatura médica mediante las siguientes bases de datos: Medline vía PubMed, Central, CINAHL, Cochrane Database of Systematic Reviews (plataforma Wiley), CUIDEN, EMBASE (Elsevier), Lilacs (Biblioteca Virtual en Salud - BVS, interfaz iAHx), SCOPUS, TESEO y WOS. La búsqueda se limitó a artículos y revisiones bibliográficas publicadas a partir de enero de 1990 hasta el mes de octubre del año 2020, en inglés y español.

La pregunta final de investigación de esta revisión se describe en la tabla 1, siguiendo el modelo PICOT, para la búsqueda digital de información en salud $(P-$ Patient/paciente; I- Intervention/intervención; $C$ Comparison/comparación; O-Outcomeldesenlace o resultado; T- Time/tiempo) (16). Los criterios de inclusión fueron: ensayos clínicos aleatorizados fase III, con disponibilidad del texto completo para su evaluación total, en donde se incluyeran mujeres mayores de 18 años, que hubiesen recibido drospirenona para el tratamiento de condiciones ginecológicas (endometriosis, síndrome de ovario poliquístico (SOP), sangrado uterino disfuncional, síndrome premenstrual y terapia de reemplazo hormonal).

Tabla 1. Pregunta de evaluación en el modelo P.I.C.O.T.

\begin{tabular}{|c|c|}
\hline $\mathrm{P}$ & $\begin{array}{l}\text { Pacientes mayores de } 18 \text { años, tratadas con drospirenona por condiciones } \\
\text { ginecológicas }\end{array}$ \\
\hline I & Drospirenona \\
\hline $\mathrm{C}$ & Anticonceptivos hormonales, placebo \\
\hline \multirow[t]{5}{*}{$\mathrm{O}$} & $\begin{array}{l}\text { Efectividad } \\
\text { Primarios } \\
\text { Tratamiento de: endometriosis, síndrome de ovario poliquístico (SOP), } \\
\text { sangrado uterino disfuncional, síndrome premenstrual y terapia de } \\
\text { reemplazo hormonal). }\end{array}$ \\
\hline & $\underline{\text { Secundarios }}$ \\
\hline & $\overline{\text { Acción a nivel endometrial }}$ \\
\hline & $\begin{array}{l}\text { Seguridad } \\
\text { Primarios } \\
\text { Efectos adversos } \\
\text { Complicaciones }\end{array}$ \\
\hline & $\begin{array}{l}\text { Secundarios } \\
\text { Cambios de humor, irritabilidad } \\
\text { Mastalgia, tensión mamaria } \\
\text { Rash, melasma } \\
\text { Proporción de sangrado }\end{array}$ \\
\hline $\mathrm{T}$ & De acuerdo a lo reportado en los estudios \\
\hline
\end{tabular}


Los criterios con los que se definió la población, fue mediante el uso de texto libre y vocabulario controlado (MeSH, Emtree y DeCS), y fueron los siguientes: "Contraceptive Agents", "Progestins" [Mesh] y "Progesterone". Los términos para las tecnologías de salud de interés que fueron asociados a través del operador booleano odds ratio (OR) fueron: "Endometriosis", "Hyperandrogenism", "Menopause", "Premenstrual Syndrome", "Uterine Hemorrhage" y "Hormone Replacement Therapy". El grupo de términos de búsqueda que definían la población, se unió a través de los términos de las tecnologías de salud de interés mediante el operador booleano "AND".

Respecto al tipo de intervención, la tecnología de interés fue drospirenona (DRSP) y las condiciones ginecológicas (endometriosis, síndrome de ovario poliquístico (SOP), sangrado uterino disfuncional, síndrome premenstrual y terapia de reemplazo hormonal). En la efectividad se consideraron como desenlaces primarios el tratamiento de la endometriosis, el síndrome de ovario poliquístico (SOP), el sangrado uterino disfuncional, el síndrome premenstrual y la terapia de reemplazo hormonal, y como resultados secundarios la acción a nivel endometrial. En la seguridad se consideraron como primarios la proporción de efectos adversos y complicaciones, y como secundarios, los cambios de humor e irritabilidad, la mastalgia y la tensión mamaria, el rash y melasma, y la proporción de sangrado.

Aspectos éticos. Al tratarse de una revisión de la literatura científica se considera como una investigación sin riesgo; de acuerdo con el artículo 11 de la Resolución 8430 de 1993 (17).

\section{RESULTADOS}

La búsqueda en las bases de datos arrojó un total de 153 referencias, al eliminar los duplicados quedaron un total de 105 referencias; de las cuales se seleccionaron 48 que cumplían criterios de inclusión según título y abstract para su posterior evaluación en texto completo.
Si bien, la nueva formulación de drospirenona con 4 mg, contiene un $33 \%$ más de ingrediente activo que un anticonceptivo oral combinado de referencia (3 $\mathrm{mg}$ drospirenona $+0,02 \mathrm{mg}$ de etinilestradiol), lo cierto es que esta exhibe un perfil farmacocinético diferente cuando se administra junto con etinilestradiol (18); sin embargo, es importante aclarar que a excepción de aquellas progestinas con actividad glucocorticoide parcial, estas per se, no aumentan la tasa de eventos trombóticos (19).

A diferencia de otras progestinas, la drospirenona tiene actividades parciales anti-mineralocorticoides, anti-estrogénicas, anti-androgénicas y antigonadotrópicas (20), contando con un perfil bioquímico y farmacológico que coincide estrechamente con el perfil de la progesterona natural (21).

En el tratamiento de la endometriosis, tanto las progestinas de acción prolongada como las de acción corta son eficaces (22); cuyo mecanismo de acción implica la decidualización y posterior atrofia del tejido endometrial, al igual que con los anticonceptivos hormonales combinados (23). La disminución del dolor, con los anticonceptivos de progestina sola, oscila entre $70 \%$ y $100 \%$, debido, en parte a las elevadas tasas de amenorrea (22).

En Japón, en el año 2015, Taniguchi et al. (24), en un grupo de mujeres de 23 a 45 años, las cuales presentaban endometrioma unilateral y dismenorrea moderada/intensa, fueron tratadas con drospirenona y etinilestradiol (DRSP/EE: $3 \mathrm{mg} / 20 \mu \mathrm{g}$ ) en régimen 24/4; estos observaron una reducción importante del diámetro del endometrioma a los 3 y 6 meses respecto del tamaño inicial, con una disminución relevante de la dismenorrea desde el primer mes y a los 3 y 6 meses. Estos autores concluyen que la terapia con $\mathrm{DRSP} / \mathrm{EE}$, es un tratamiento prometedor no solo para reducir el tamaño del endometrioma sino también para el manejo de la dismenorrea.

La drospirenona, en mujeres que tienen síndrome de ovario poliquístico (SOP), ha demostrado ser eficaz para disminuir el hirsutismo y los niveles de 
testosterona, además de aumentar los niveles de globulina transportadora de hormonas sexuales (SHBG) (25, 26). La combinación DRSP/EE, demostró beneficios en los signos clínicos y bioquímicos del hiperandrogenismo, gracias a su actividad anti-androgénica; sin evidencia de deterioro en los parámetros metabólicos, secundario a su actividad anti-mineralocorticoide (27). En relación con la mejoría del acné (leve y moderado), la drospirenona demostró un efecto similar o superior a otras progestinas (28).

En el síndrome premenstrual (SPM) y el trastorno disfórico premenstrual (TDPM) (una forma grave de SPM); la DRSP/EE (3 mg/20 $\mu \mathrm{g})$, ayuda a tratar los síntomas premenstruales (29). La Food and Drug Administration (FDA), aprobó esta combinación, en el régimen 24/4, para el tratamiento de los síntomas emocionales y físicos del TDPM $(30,31)$.

El tratamiento médico ha de considerarse como la primera línea terapéutica, en el manejo del sangrado uterino disfuncional, no estructural. Las progestinas son útiles en pacientes que desean el efecto anticonceptivo, pero que no son candidatas a la terapia hormonal combinada, y que presentan ciclos irregulares y anovulatorios (32) debido a que promueven la atrofia endometrial por diversos mecanismos (33).

La terapia combinada de $2 \mathrm{mg}$ de drospirenona/ $1 \mathrm{mg}$ $17 \beta$-estradiol $\left(E_{2}\right)$, ha demostrado ser eficaz para aliviar los síntomas de la menopausia y la prevención de la osteoporosis posmenopáusica (34); con un efecto antihipertensivo significativo (35).

Respecto a los efectos adversos de las progestinas, se incluyen cambios en el peso, sangrado intermenstrual, aumento de la actividad de la migraña, amenorrea, cambios de humor/depresión, náuseas y sensibilidad en las mamas (36).

\section{DISCUSIÓN}

La drospirenona es la progestina sintética más similar a la progesterona natural, y estructuralmente semejante a la espironolactona, actuando como un antagonista de los receptores de aldosterona con actividad anti-mineralocorticoide clínicamente reconocida (37).

Se ha demostrado, durante más de 50 años, que las progestinas son eficaces en el tratamiento de la endometriosis, siendo el principio fundamental inducir la amenorrea (38), ya que reducen o eliminan los síntomas y el dolor pélvico en cerca del $90 \%$ de las pacientes; de igual forma, tienen indicaciones en otras condiciones ginecológicas.

La eficacia de las progestinas, no solo se relaciona con sus acciones inhibidoras del crecimiento endometrial, sino también con la inducción de la anovulación, inhibición del crecimiento de vasos sanguíneos y acciones antiinflamatorias (39). Las cuales se pueden administrar en preparaciones orales, inyecciones, implantes subdérmicos $\mathrm{y}$ sistemas intrauterinos.

La actividad anti-androgénica de la drospirenona, le permite que sea usada como tratamiento complementario del hirsutismo o ser de elección en mujeres que se quejan de crecimiento excesivo del cabello, además de disminuir la gravedad del acné; demostrando ser efectiva para reducir la hiperandrogenemia $(25,27,40)$. Estas características podrían hacerla más efectiva para el tratamiento del SOP que otras progestinas.

Los anticonceptivos hormonales combinados que contienen una dosis baja de etinilestradiol y drospirenona, han demostrado ser eficaces para tratar el trastorno disfórico premenstrual (TDPM) $(31,41)$, ya que, al acortar el intervalo sin píldoras de 7 a 4 días, proporciona una mejor supresión de la producción de esteroides endógenos; además las propiedades anti-mineralcorticoides únicas de la drospirenona, generan un superior beneficio en la reducción de los síntomas del TDPM, proporcionando una mayor rebaja en la gravedad $(42,43)$.

La drospirenona disminuye el riesgo de hiperplasia endometrial, en comparación con los estrógenos sin oposición (44). En el sangrado uterino disfuncional 
agudo, el manejo hormonal es la terapia médica de primera línea $(45,46)$; sin embargo, no hay consistencia entre los regímenes hormonales recomendados y muy poca o ninguna evidencia científica de eficacia para cualquiera de ellos. El uso de anticonceptivos hormonales combinados con 30 $\mu \mathrm{g}$ o $50 \mu \mathrm{g}$ de etinilestradiol en combinación con cualquier progestina, es una opción de tratamiento en el sangrado uterino disfuncional, en mujeres sin trastornos hemorrágicos subyacentes (46).

La drospirenona en combinación con un estrógeno es eficaz para minimizar la incidencia y la gravedad de los sofocos en mujeres posmenopáusicas $(47,48)$; contándose con la información disponible respecto de su seguridad y tolerabilidad.

Muchos estudios han evaluado los efectos adversos de los anticonceptivos hormonales orales que contienen drospirenona; los cuales incluyen la actividad anti-androgénica y anti-mineralocorticoide de esta progestina $(1,25,30,34)$.

La drospirenona, a la luz de la evidencia disponible, en la presente revisión se justifica más allá de su utilización como anticonceptivo; lo cual la ubica, sino en la primera línea de elección para el tratamiento de condiciones ginecológicas, y dada su adecuada tolerancia, bien podría considerarse una alternativa de segunda línea en mujeres que presentan efectos adversos a los fármacos de primera elección, o en aquellas en que se presente una falla en el tratamiento de primera línea o tengan contraindicación a su uso.

Los hallazgos de las publicaciones aquí citadas, hacen reflexionar que la drospirenona es una eventual alternativa para tratar condiciones ginecológicas frecuentes en la consulta; la cual debería ser tenida en cuenta para ofrecerla a las mujeres en las cuales se contraindica la anticoncepción hormonal combinada, o en las que no la toleran, en especialmente en las mujeres que experimentan síntomas y que no desean quedar embarazadas.
No se conoce si la drospirenona de $4 \mathrm{mg}$ en el régimen de 24/4, sea efectiva para tratar a las mujeres con situaciones ginecológicas distintas a la anticoncepción, o si son iguales o mejores que los anticonceptivos hormonales combinados; por lo tanto, se necesitan ensayos clínicos más grandes y con mayor calidad estadística para evaluar estos aspectos.

\section{CONCLUSIONES}

La drospirenona presenta efectividad y seguridad en el tratamiento de condiciones ginecológicas frecuentes (endometriosis, síndrome de ovario poliquístico (SOP), sangrado uterino disfuncional, síndrome premenstrual y en la terapia de reemplazo hormonal), más allá de su acción como anticonceptivo; con una baja frecuencia de efectos adversos.

Se hacen necesarios estudios que evalúen el régimen 24/4 de la drospirenona a fin de evaluar si este es igual de efectivo a la combinación de anticonceptivos hormonales orales, en el tratamiento de las condiciones ginecológicas descritas.

CONTRIBUCIONES DE LOS AUTORES: FED: concepción y diseño del estudio, recolección y análisis de resultados, redacción del artículo, revisión crítica y aprobación de versión final, responsable de la veracidad e integridad del artículo.

\section{AGRADECIMIENTOS}

A los directivos de Hathor, Clínica Sexológica, por su permanente e incondicional apoyo. A la doctora Lilian Orozco Santiago, en tributo a su amor y acompañamiento, su gestión hizo posible que este humilde ser aún continúe con vida.

FINANCIACIÓN: este estudio fue financiado con recursos propios del autor. No hubo intervención de la industria farmacéutica.

CONFLICTOS DE INTERÉS: ninguno que declarar. 


\section{REFERENCIAS}

1. Oelkers W. Drospirenone, a progestogen with antimineralocorticoid properties: a short review. Mol Cell Endocrinol. 2004 Mar 31; 217(1-2): 255-61. https://doi.org/10.1016/j.mce.2003.10.030

2. Oelkers WH. Drospirenone in combination with estrogens: for contraception and hormone replacement therapy. Climacteric. 2005 Oct;8 Suppl 3: 19-27. https://doi.org/10.1080/13697130500330341

3. Stanczyk FZ. All progestins are not created equal. Steroids. 2003 Nov; 68(10-13): 879-90. https://doi.org/10.1016/j.steroids.2003.08.003

4. Stacy RA, Nelson AL. A new progestogen-only medical therapy for outpatient management of acute, abnormal uterine bleeding: a pilot sudy. Am J Obstet Gynecol. 2013; 208(6): 499.e1-5. https://doi.org/10.1016/j.ajog.2013.02.013

5. Bhatia SC, Bhatia SK. Diagnosis and treatment of premenstrual dysphoric disorder. Am Fam Physician. 2002 Oct 1; 66(7): 1239-48.

6. Raudrant D, Rabe T. Progestogens with antiandrogenic properties. Drugs. 2003; 63(5): 463-92. https://doi.org/10.2165/00003495-200363050-00003

7. Benagiano G, Pera A, Primiero FM. The endometrium and hormonal contraceptives. Hum. Reprod. 2000; 15(1): $101-18$

8. Deligdisch L. Hormonal pathology of the endometrium. Mod Pathol. 2000 Mar; 13(3): 285-94. https://doi.org/10.1038/modpathol.3880050

9. Elger W, Beier S, Pollow K, Garfield R, Shi SQ, Hillisch A. Conception and pharmacodynamic profile of drospirenone. Steroids. 2003 Nov; 68(10-13): 891-905. https://doi.org/10.1016/j.steroids.2003.08.008

10. Duijkers IJ, Heger-Mahn D, Drouin D, Skouby S. A randomised study comparing the effect on ovarian activity of a progestogen-only pill (POP) containing desogestrel and a new POP containing drospirenone in a 24/4 regimen. Eur J Contracept Reprod Health Care. 2015; 20(6): 419-27. https://doi.org/10.3109/13625187.2015.1044082

11. Archer DF, Ahrendt HJ, Drouin D. Drospirenone-only oral contraceptive: results from a multicenter noncomparative trial of efficacy, safety and tolerability. Contraception. 2015 Nov; 92(5): 439-44. https://doi.org/10.1016/j.contraception.2015.07.014

12. Duijkers IJM, Heger-Mahn D, Drouin D, Colli E, Skouby S. Maintenance of ovulation inhibition with a new progestogen-only pill containing drospirenone after scheduled 24-h delays in pill intake. Contraception. 2016 Apr; 93(4): 303-309. https://doi.org/10.1016/j.contraception.2015.12.007

13. Palacios S, Colli E, Regidor PA. Multicenter, phase III trials on the contraceptive efficacy, tolerability and safety of a new drospirenone-only pill. Acta Obstet Gynecol Scand. 2019 Dec; 98(12): 1549-1557. https://doi.org/10.1111/aogs.13688

14. Roach RE, Helmerhorst FM, Lijfering WM, Stijnen T, Algra A, Dekkers OM. Combined oral contraceptives: the risk of myocardial infarction and ischemic stroke. Cochrane Database Syst Rev. 2015 Aug 27; 2015(8): CD011054. https://doi.org/10.1002/14651858.CD011054.pub2

15. Stegeman $B H$, de Bastos $M$, Rosendaal FR, van Hylckama Vlieg A, Helmerhorst FM, Stijnen T, Dekkers OM. Different combined oral contraceptives and the risk of venous thrombosis: systematic review and network meta-analysis. BMJ. 2013 Sep 12; 347: f5298. https://doi.org/10.1136/bmj.f5298

16. Rios LP, Ye C, Thabane L. Association between framing of the research question using the PICOT format and reporting quality of randomized controlled trials. $B M C$ Med Res Methodol. 2010 Feb 5; 10:11. https://doi.org/10.1186/1471-2288-10-11

17. Ministerio de Salud y Protección Social. Resolución 8430 de 1993.

18. Richter WH, Koytchev R, Kirkov V, Merki G, Colli E, Regidor PA. Comparative pharmacokinetic estimates of drospirenone alone and in combination with ethinyl estradiol after single and repeated oral administration in healthy females. Contraception. 2020 Feb; 101(2): 137143. https://doi.org/10.1016/j.contraception.2019.10.005

19. Schindler AE. Non-contraceptive benefits of oral hormonal contraceptives. Int J Endocrinol Metab. 2013 Winter; 11(1): 41-7. https://doi.org/10.5812/ijem.4158

20. Regidor PA. The clinical relevance of progestogens in hormonal contraception: Present status and future 
developments. Oncotarget. 2018 Oct 2; 9(77): 3462834638. https://doi.org/10.18632/oncotarget.26015

21. White WB, Hanes V, Chauhan V, Pitt B. Effects of a new hormone therapy, drospirenone and 17-betaestradiol, in postmenopausal women with hypertension. Hypertension. 2006 Aug; 48(2): 246-53. https://doi.org/10.1161/01.HYP.0000232179.60442.84

22. Kodaman PH. Current strategies for endometriosis management. Obstet Gynecol Clin North Am. 2015 Mar; 42(1): 87-101. https://doi.org/10.1016/j.ogc.2014.10.005

23. Practice Committee of the American Society for Reproductive Medicine. Treatment of pelvic pain associated with endometriosis: a committee opinion. Fertil Steril. 2014 Apr; 101(4): 927-35. https://doi.org/10.1016/j.fertnstert.2014.02.012

24. Taniguchi F, Enatsu A, Ota I, Toda T, Arata K, Harada T. Effects of low dose oral contraceptive pill containing drospirenone/ethinylestradiol in patients with endometrioma. Eur J Obstet Gynecol Reprod Biol. 2015; 191:

https://doi.org/10.1016/j.ejogrb.2015.06.006

$116-20$

25. Pehlivanov B, Mitkov M. Efficacy of an oral contraceptive containing drospirenone in the treatment of women with polycystic ovary syndrome. Eur $J$ Contracept Reprod Health Care. 2007 Mar; 12(1): 30-5. https://doi.org/10.1080/13625180600983082

26. Batukan C, Muderris II. Efficacy of a new oral contraceptive containing drospirenone and ethinyl estradiol in the long-term treatment of hirsutism. Fertil Steril. $2006 \quad$ Feb; 85(2): 436-40. https://doi.org/10.1016/j.fertnstert.2005.07.1311

27. Guido M, Romualdi D, Giuliani M, Suriano R, Selvaggi L, Apa R, Lanzone A. Drospirenone for the treatment of hirsute women with polycystic ovary syndrome: a clinical, endocrinological, metabolic pilot study. J Clin Endocrinol Metab. 2004 Jun; 89(6): 2817-23. https://doi.org/10.1210/jc.2003-031158

28. van Vloten WA, van Haselen CW, van Zuuren EJ, Gerlinger C, Heithecker R. The effect of 2 combined oral Contraceptives containing either drospirenone or cyproterone acetate on acne and seborrhea. Cutis. 2002 Apr; 69(4 Suppl): 2-15.

29. Lopez LM, Kaptein AA, Helmerhorst FM. Oral contraceptives containing drospirenone for premenstrual syndrome. Cochrane Database of Systematic Reviews 2008, Issue 1. Art. No.: CD006586. https://doi.org/10.1002/14651858.CD006586.pub2

30. Pearlstein TB, Bachmann GA, Zacur HA, Yonkers KA. Treatment of premenstrual dysphoric disorder with a new drospirenone-containing oral contraceptive formulation. Contraception. 2005 Dec; 72(6): 414-21. https://doi.org/10.1016/j.contraception.2005.08.021

31. Yonkers KA, Brown C, Pearlstein TB, Foegh M, Sampson-Landers C, Rapkin A. Efficacy of a new lowdose oral contraceptive with drospirenone in premenstrual dysphoric disorder. Obstet Gynecol. 2005 Sep; 106(3): 492-501. https://doi.org/10.1097/01.AOG.0000175834.77215.2e

32. Farrukh JB, Towriss K, McKee N. Abnormal uterine bleeding: Taking the stress out of controlling the flow. Can Fam Physician. 2015 Aug; 61(8): 693-7.

33. Bradley LD, Gueye NA. The medical management of abnormal uterine bleeding in reproductive-aged women. Am J Obstet Gynecol. 2016 Jan; 214(1): 31-44. https://doi.org/10.1016/j.ajog.2015.07.044

34. Foidart JM, Faustmann T. Advances in hormone replacement therapy: weight benefits of drospirenone, a 17alpha-spirolactone-derived progestogen. Gynecol Endocrinol. 2007 Dec; 23(12): 692-9. https://doi.org/10.1080/09513590701582323

35. Preston RA, Alonso A, Panzitta D, Zhang P, Karara AH. Additive effect of drospirenone/17-beta-estradiol in hypertensive postmenopausal women receiving enalapril. Am J Hypertens. 2002 Sep; 15(9): 816-22. https://doi.org/10.1016/s0895-7061(02)02980-1

36. Bitzer J, Amy JJ, Beerthuizen R, Birkhäuser M, Bombas $\mathrm{T}$, Creinin M, et al. Statement on combined hormonal contraceptives containing third- or fourth-generation progestogens or cyproterone acetate, and the associated risk of thromboembolism. Eur J Contracept Reprod Health Care. 2013 Jun; 18(3): 143-7. https://doi.org/10.3109/13625187.2013.792637

37. Krattenmacher R. Drospirenone: pharmacology and pharmacokinetics of a unique progestogen. Contraception. $2000 \quad \mathrm{Jul} ; \quad 62(1)$ : 29-38. https://doi.org/10.1016/s0010-7824(00)00133-5

38. Schweppe KW. The current place of progestins in the treatment of endometriosis. Expert Review of Obstetrics 
\& Gynecology. 2012; 7(2):141-18. https://doi.org/10.1586/eog.11.71

39. Fainaru O, Linda C. Giudice, Johannes L. H. Evers, David L. Healy (eds): Endometriosis: Science and Practice: Wiley-Blackwell, Oxford, 2012, 600 pp, ISBN 978-1-4443-3213-1. J Obstet Gynaecol India. 2014 Jun; 64(3): 224-5. https://doi.org/10.1007/s13224-014-0565$\underline{9}$

40. Mathur R, Levin O, Azziz R. Use of ethinylestradiol/drospirenone combination in patients with the polycystic ovary syndrome. Ther Clin Risk Manag. $2008 \quad$ Apr; 487): 487-92. https://doi.org/10.2147/tcrm.s6864

41. Rapkin AJ, McDonald M, Winer SA. Ethinyl estradiol/drospirenone for the treatment of the emotional and physical symptoms of premenstrual dysphoric disorder. Womens Health (Lond). 2007 Jul; 3(4): 395408. https://doi.org/10.2217/17455057.3.4.395

42. Foidart JM, Wuttke W, Bouw GM, Gerlinger C, Heithecker R. A comparative investigation of contraceptive reliability, cycle control and tolerance of two monophasic oral contraceptives containing either drospirenone or desogestrel. Eur J Contracept Reprod Health Care. 2000 Jun; 5(2): 124-34. https://doi.org/10.1080/13625180008500387

43. Sangthawan M, Taneepanichskul S. A comparative study of monophasic oral contraceptives containing either drospirenone $3 \mathrm{mg}$ or levonorgestrel 150 microg on premenstrual symptoms. Contraception. 2005 Jan; 71(1): 1-7. https://doi.org/10.1016/j.contraception.2004.07.010

44. Archer DF. Drospirenone and estradiol: a new option for the postmenopausal woman. Climacteric. 2007 Feb;10 Suppl 1: 3-10. https://doi.org/10.1080/13697130601114859

45. James AH, Kouides PA, Abdul-Kadir R, Dietrich JE, Edlund $\mathrm{M}$, Federici $\mathrm{AB}$, et al. Evaluation and management of acute menorrhagia in women with and without underlying bleeding disorders: consensus from an international expert panel. Eur J Obstet Gynecol Reprod Biol. 2011 Oct; 158(2): 124-34. https://doi.org/10.1016/j.ejogrb.2011.04.025

46. American College of Obstetricians and Gynecologists. Committee Opinion no. 557: Management of acute abnormal uterine bleeding in nonpregnant reproductiveaged women. Obstet Gynecol. 2013; 121(4): 891-896.

47. Lee BS, Kang BM, Yoon BK, Choi H, Park HM, Kim JG. Efficacy and tolerability of estradiol $1 \mathrm{mg}$ and drospirenone $2 \mathrm{mg}$ in postmenopausal Korean women: a double-blind, randomized, placebo-controlled, multicenter study. Maturitas. 2007 Aug 20; 57(4): 361-9. https://doi.org/10.1016/j.maturitas.2007.03.004

48. Chaikittisilpa S, Angsuwathana S, Chaovisitsaree S, Jirapinyo M, Wilawan K, Panyakhamlerd K, et al. Clinical responses to the combination of estradiol and drospirenone in symptomatic postmenopausal Thai women. J. Med. Assoc. Thai. 2011, 94, 1019-1025. 